\title{
Exponential Sums with Farey Fractions
}

by

\author{
Igor E. SHPARLINSKI
}

Presented by Jerzy KACZOROWSKI

Summary. For positive integers $m$ and $N$, we estimate the rational exponential sums with denominator $m$ over the reductions modulo $m$ of elements of the set

$$
\mathcal{F}(N)=\{s / r: r, s \in \mathbb{Z}, \operatorname{gcd}(r, s)=1, N \geq r>s \geq 1\}
$$

of Farey fractions of order $N$ (only fractions $s / r$ with $\operatorname{gcd}(r, m)=1$ are considered).

1. Introduction. For any integer $N \geq 1$, we consider the set

$$
\mathcal{F}(N)=\{s / r: r, s \in \mathbb{Z}, \operatorname{gcd}(r, s)=1, N \geq r>s \geq 1\}
$$

of Farey fractions of order $N$. There is an extensive literature where various distributional properties of Farey fractions are investigated. In particular, some of these properties are directly related to the Riemann Hypothesis. (See, for example, the survey [4] as well as the more recent works $[1,2,3,7,8]$ and references therein.)

Here we consider an apparently new problem of the distribution of $\mathcal{F}(N)$ in residue classes modulo an integer $m \geq 2$. In particular, we show that for any interval $\mathcal{I}=[k, k+h-1] \subseteq[0, m-1]$ and any integer $N \geq m^{\varepsilon}$, where $\varepsilon>0$ is fixed, the number $R_{m}(N, \mathcal{I})$ of Farey fractions $s / r \in \mathcal{F}(N)$ with $\operatorname{gcd}(r, m)=1$ and such that $s / r \equiv z(\bmod m)$ for some $z \in \mathcal{I}$ is close to its expected value, provided that $m$ is large enough.

Naturally, our main tool is bounds for exponential sums. For any integer $m \geq 2$ and any real $z$ we put

$$
\mathbf{e}_{m}(z)=\exp (2 \pi i z / m)
$$


and consider the exponential sums

$$
S_{m}(a ; N)=\sum_{\substack{s / r \in \mathcal{F}(N) \\ \operatorname{gcd}(r, m)=1}} \mathbf{e}_{m}(a s / r), \quad a \in \mathbb{Z} .
$$

(Note that each fraction $s / r \in \mathcal{F}(N)$ is reduced modulo $m$ before it is used in the sum $S_{m}(a ; N)$.)

We remark that for larger values of $N$, namely for $N \geq m^{1 / 2+\varepsilon}$, one can use the results in $[12,13]$ to show that almost all residue classes from the reduced residue system modulo $m$ are represented by the elements of $\mathcal{F}(N)$ asymptotically the same number of times. A variant of such a result is also given in [5].

We write $V=U^{o(1)}$ to indicate the quantity which satisfies

$$
\lim _{U \rightarrow \infty} \frac{\log V}{\log U}=0 .
$$

TheOREM 1. For any integer $a \not \equiv 0(\bmod m)$,

$$
\left|S_{m}(a ; N)\right| \leq N(N m)^{o(1)} \quad \text { as } N, m \rightarrow \infty .
$$

Combining Theorem 1 with some standard technique, we also derive the following asymptotic formula for $R_{m}(N, \mathcal{I})$.

ThEOREM 2. Uniformly over the intervals $\mathcal{I}=[k, k+h-1] \subseteq[0, m-1]$,

$$
\left|R_{m}(N, \mathcal{I})-\frac{6}{\pi^{2}} \prod_{p \mid m}\left(1+\frac{1}{p}\right)^{-1} \frac{h}{m} N^{2}\right| \leq N(N m)^{o(1)}
$$

as $N, m \rightarrow \infty$, where the product is taken over all primes $p \mid m$.

2. Double sums. Here we obtain a rather general statement which may be of independent interest.

We use the notations $U=O(V)$ and $U \ll V$ to indicate that $|U| \leq c V$ for some absolute constant $c>0$.

Lemma 3. Let $Y \geq 1$ be an arbitrary integer. Assume that for each integer $x$ we are given two integers $L_{x}$ and $U_{x}$ with $0 \leq L_{x}<m$ and $U_{x} \leq Y$. Then for any integer $a \not \equiv 0(\bmod m)$,

$$
\left|\sum_{\substack{x=1 \\ \operatorname{gcd}(x, m)=1}}^{X} \sum_{y=L_{x}+1}^{U_{x}} \mathbf{e}_{m}(a y / x)\right| \leq(X+Y)(X m)^{o(1)}
$$

as $X, m \rightarrow \infty$. 
Proof. Let

$$
W=\sum_{\substack{x=1 \\ \operatorname{gcd}(x, m)=1}}^{X} \sum_{y=L_{x}+1}^{U_{x}} \mathbf{e}_{m}(a y / x) .
$$

We may assume that

$$
0<a \leq m-1 .
$$

For a rational number $\alpha=u / v$ with $\operatorname{gcd}(v, m)=1$, we denote by $\rho(\alpha)$ the unique integer $w$ with

$$
w \equiv u / v \quad(\bmod m) \quad \text { and } \quad-m / 2<w<(m+1) / 2 .
$$

Using the bound

$$
\sum_{y=L+1}^{L+H} \mathbf{e}_{m}(\alpha y) \ll \min \left\{H, \frac{m}{|\rho(\alpha)|}\right\},
$$

which holds for any rational $\alpha$ and integers $L$ and $H$ (see [10, Bound (8.6)]), we obtain

$$
W \ll \sum_{\substack{x=1 \\ \operatorname{gcd}(x, m)=1}}^{X} \min \left\{Y, \frac{m}{\rho(a / x)}\right\} .
$$

We now put $J=\lceil\log (m / 2)\rceil$ and define the sets

$$
\mathcal{X}_{j}=\left\{x: 1 \leq x \leq X, \operatorname{gcd}(x, m)=1, e^{j} \leq|\rho(a / x)|<e^{j+1}\right\},
$$

where $j=0, \ldots, J$. Therefore

$$
W \ll \sum_{j=0}^{J} \# \mathcal{X}_{j} \min \left\{Y, \frac{m}{e^{j}}\right\} \ll Y \sum_{j=0}^{J_{0}} \# \mathcal{X}_{j}+m \sum_{j=J_{0}+1}^{J} \# \mathcal{X}_{j} e^{-j},
$$

where $J_{0}$ is the largest $j \leq J$ with $e^{j} \leq m / Y$.

To estimate $\# \mathcal{X}_{j}$ we note that if $e^{j} \leq|\rho(a / x)|<e^{j+1}$, then $x z \equiv a$ $(\bmod m)$ for some integer $z$ with $0<|z|<e^{j+1}$. Thus $x z=a+m k$ for some integer $k$ with $|k|<e^{j+1} X / m+1$. Hence there are at most $O\left(e^{j} X / m+1\right)$ possible values of $k$ and for each fixed $k \ll e^{j} X / m$ there are

$$
\tau(|a+m k|)=(X m)^{o(1)}
$$

nonzero integers $x$ and $z$ with $x z=a+m k$, where $\tau(u)$ is the number of positive integer divisors of an integer $u \neq 0$ (see [9, Theorem 317]). Therefore we obtain the estimate

$$
\# \mathcal{X}_{j} \leq\left(e^{j} X / m+1\right) m^{o(1)}
$$


which after inserting into (1) gives

$$
|W| \leq Y \sum_{j=0}^{J_{0}}\left(e^{j} X / m+1\right) m^{o(1)}+m^{1+o(1)} \sum_{j=J_{0}+1}^{J}\left(e^{j} X / m+1\right) e^{-j} .
$$

We now have

$$
\sum_{j=0}^{J_{0}}\left(e^{j} X / m+1\right) \ll e^{J_{0}} X / m+J_{0} \ll X / Y+m^{o(1)}
$$

and also

$$
\sum_{j=J_{0}+1}^{J}\left(e^{j} X / m+1\right) e^{-j} \leq J X / m+e^{-J_{0}} \leq X m^{-1+o(1)}+Y / m .
$$

Substituting the above bounds into (2), we obtain the desired result.

3. Proof of Theorem 1. For an integer $d \geq 1$ we use $\mu(d)$ to denote the Möbius function. We recall that $\mu(1)=1, \mu(d)=0$ if $d \geq 2$ is not square-free, and $\mu(d)=(-1)^{\omega(d)}$ otherwise, where $\omega(d)$ is the number of prime divisors of $d$. Then by the inclusion-exclusion principle,

$$
\begin{aligned}
S_{m}(a ; N) & =\sum_{d=1}^{N} \mu(d) \sum_{\substack{r=1 \\
\operatorname{gcd}(r, m)=1 \\
d \mid r}}^{N} \sum_{\substack{s=r+1 \\
d \mid s}} \mathbf{e}_{m}(a s / r) \\
& =\sum_{d=1}^{N} \mu(d) \sum_{\substack{x=1 \\
\operatorname{gcd}(x, m)=1}}^{\lfloor N / d\rfloor} \sum_{y=x+1}^{\lfloor N / d\rfloor} \mathbf{e}_{m}(a y / x) .
\end{aligned}
$$

Now, for each $d=1, \ldots, N$ we apply Lemma 3 with $L_{x}=x$ and $U_{x}=\lfloor N / d\rfloor$, and after a short calculation we obtain the desired result.

4. Proof of Theorem 2. For any integer $N \geq 1$, let

$$
\mathcal{F}_{m}(N)=\{s / r \in \mathcal{F}(N): \operatorname{gcd}(r, m)=1\} .
$$

Now using the Erdös-Turán inequality (see [6, 11]), which links the discrepancy with exponential sums, we immediately deduce from Theorem 1 that

$$
\left|R_{m}(N, \mathcal{I})-\frac{h}{m} \# \mathcal{F}_{m}(N)\right| \leq N^{1+o(1)} m^{o(1)} .
$$

It remains to approximate $\# \mathcal{F}_{m}(N)$. We follow the proof of the well-known asymptotic formula for $\# \mathcal{F}(N)$ given in [9, Theorem 330]. 
We have

$$
\# \mathcal{F}_{m}(N)=\sum_{\substack{r=1 \\ \operatorname{gcd}(r, m)=1}}^{N} \varphi(r)=\sum_{\substack{r=1 \\ \operatorname{gcd}(r, m)=1}}^{N} r \sum_{d \mid r} \frac{\mu(d)}{d},
$$

where $\varphi(r)$ is the Euler function (see [9, Equation (16.3.1)]). Interchanging the order of summation, we obtain

$$
\# \mathcal{F}_{m}(N)=\sum_{\substack{r=1 \\ \operatorname{gcd}(r, m)=1}}^{N} \varphi(r)=\sum_{\substack{d=1 \\ \operatorname{gcd}(d, m)=1}}^{N} \frac{\mu(d)}{d} \sum_{\substack{r=1 \\ \operatorname{gcd}(r, m)=1 \\ r \equiv 0(\bmod d)}}^{N} r .
$$

Then replacing $r$ with $d t$, we deduce

$$
\# \mathcal{F}_{m}(N)=\sum_{\substack{d=1 \\ \operatorname{gcd}(d, m)=1}}^{N} \mu(d) \sum_{\substack{1 \leq t \leq N / d \\ \operatorname{gcd}(t, m)=1}}^{N} t .
$$

We note that by the inclusion-exlusion principle, for any real $T$,

$$
\begin{aligned}
\sum_{\substack{1 \leq t \leq T \\
\operatorname{gcd}(t, m)=1}} t & =\sum_{e \mid m} \mu(e) \sum_{\substack{1 \leq t \leq T \\
e \mid t}}^{N} t=\sum_{e \mid m} \mu(e) e \sum_{1 \leq u \leq T / e}^{N} u \\
& =\sum_{e \mid m} \mu(e) e\left(\frac{T^{2}}{2 e^{2}}+O(T / e)\right)=\frac{1}{2} T^{2} \sum_{e \mid m} \frac{\mu(e)}{e}+O(T \tau(m)) .
\end{aligned}
$$

Using [9, Theorem 317 and Equation (16.3.1)], we obtain

$$
\sum_{\substack{1 \leq t \leq T \\ \operatorname{gcd}(t, m)=1}} t=\frac{\varphi(m)}{2 m} T^{2}+O\left(T m^{o(1)}\right),
$$

which after substitution into (3) yields

$$
\begin{aligned}
\# \mathcal{F}_{m}(N) & =\sum_{\substack{d=1 \\
\operatorname{gcd}(d, m)=1}}^{N} \mu(d)\left(\frac{\varphi(m)}{m} \cdot \frac{N}{2 d^{2}}+O\left(\frac{N m^{o(1)}}{d}\right)\right) \\
& =\frac{\varphi(m)}{2 m} N^{2} \sum_{\substack{d=1 \\
\operatorname{gcd}(d, m)=1}}^{N} \frac{\mu(d)}{d^{2}}+O\left(N m^{o(1)}\right) \\
& =\frac{\varphi(m)}{2 m} N^{2} \sum_{\substack{d=1 \\
\operatorname{gcd}(d, m)=1}}^{\infty} \frac{\mu(d)}{d^{2}}+O\left(N m^{o(1)}\right) .
\end{aligned}
$$


Obviously

$$
\sum_{\substack{d=1 \\ \operatorname{cod}(d, m)=1}}^{\infty} \frac{\mu(d)}{d^{2}}=\prod_{p \nmid m}\left(1-\frac{1}{p^{2}}\right)=\frac{m}{\varphi(m)} \prod_{p \mid m}\left(1+\frac{1}{p}\right)^{-1} \prod_{p}\left(1-\frac{1}{p^{2}}\right),
$$

where the product is taken over all primes $p$ (see [9, Theorem 285]). Then recalling that

$$
\prod_{p}\left(1-\frac{1}{p^{2}}\right)=\sum_{\substack{d=1 \\ \operatorname{gcd}(d, m)=1}}^{\infty} \frac{\mu(d)}{d^{2}}=\zeta(2)^{-1}=\frac{6}{\pi^{2}}
$$

(see [9, Theorem 287 and Equation (17.2.2)]), we obtain

$$
\# \mathcal{F}_{m}(N)=\frac{6}{\pi^{2}} \prod_{p \mid m}\left(1+\frac{1}{p}\right)^{-1} N^{2}+O\left(N m^{o(1)}\right) .
$$

This completes the proof of the theorem.

Acknowledgements. The author would like thank Andrew Ledoan for a very careful reading and numerous comments on the original version of this paper.

During the preparation of this paper, the author was supported in part by ARC grant DP0556431.

\section{References}

[1] E. Alkan, A. H. Ledoan, M. Vâjâitu and A. Zaharescu, Discrepancy of fractions with divisibility constraints, Monatsh. Math. 149 (2006), 179-192.

[2] E. Alkan, M. Xiong and A. Zaharescu, Quotients of values of the Dedekind eta function, Math. Ann. 342 (2008), 157-176.

[3] R. Balasubramanian, S. Kanemitsu and M. Yoshimoto, Euler products, Farey series, and the Riemann hypothesis, Publ. Math. Debrecen 69 (2006), 1-16.

[4] C. Cobeli and A. Zaharescu, The Haros-Farey sequence at two hundred years, Proc. Acta Univ. Apulensis Math. Inform. 5 (2003), 1-38.

[5] A. C. Cojocaru and I. E. Shparlinski, Distribution of Farey fractions in residue classes and Lang-Trotter conjectures on average, Proc. Amer. Math. Soc. 136 (2008), 1977-1986.

[6] M. Drmota and R. Tichy, Sequences, Discrepancies and Applications, Springer, Berlin, 1997.

[7] A. Fujii, On the Farey series and the Riemann hypothesis, Comment. Math. Univ. St. Pauli 54 (2005), 211-235.

[8] -, On the Farey series and the Hecke L-functions, ibid. 56 (2007), 97-162.

[9] G. H. Hardy and E. M. Wright, An Introduction to the Theory of Numbers, Oxford Univ. Press, Oxford, 1979.

[10] H. Iwaniec and E. Kowalski, Analytic Number Theory, Amer. Math. Soc., Providence, RI, 2004. 
[11] L. Kuipers and H. Niederreiter, Uniform Distribution of Sequences, Wiley-Interscience Publ., 1974.

[12] I. E. Shparlinski, Primitive points on a modular hyperbola, Bull. Polish Acad. Sci. Math. 54 (2006), 193-200.

[13] - Distribution of inverses and multiples of small integers and the Sato-Tate conjecture on average, Michigan Math. J. 56 (2008), 99-111.

Igor E. Shparlinski

Department of Computing

Macquarie University

Sydney, NSW 2109, Australia

E-mail: igor@ics.mq.edu.au

Received February 4, 2009;

received in final form June 15, 2009 